Proyecciones Journal of Mathematics

Vol. 41, No 1, pp. 319-334, February 2022.

Universidad Católica del Norte

Antofagasta - Chile

\title{
Local vertex antimagic chromatic number of some wheel related graphs
}

\author{
R. Shankar \\ Vellore Institute of Technology, India \\ and \\ M. Nalliah \\ Vellore Institute of Technology, India \\ Received: August 2020. Accepted : May 2021
}

\begin{abstract}
Let $G=(V, E)$ be a graph of order $p$ and size $q$ having no isolated vertices. A bijection $f: E \rightarrow\{1,2,3, \ldots, q\}$ is called a local antimagic labeling if for all $u v \in E$ we have $w(u) \neq w(v)$, the weight $w(u)=\sum_{e \in E(u)} f(e)$, where $E(u)$ is the set of edges incident to $u$. A graph $G$ is local antimagic if $G$ has a local antimagic labeling. The local antimagic chromatic number $\chi_{l a}(G)$ is defined to be the minimum number of colors taken over all colorings of $G$ induced by local antimagic labelings of $G$. In this paper, we determine the local antimagic chromatic number for some wheel related graphs.
\end{abstract}

Keywords: Local antimagic labeling, Local antimagic chromatic number, Helm graph.

2020 Mathematics Subject Classification: $05 C 78,05 C 15$. 


\section{Introduction}

The graph $G=(V, E)$ we mean a finite, undirected graph with neither loops nor multiple edges. The order and size of $G$ are denoted by $|V|=p$ and $|E|=q$ respectively. For graph-theoretic terminology, we refer to Chartrand and Lesniak [4].

Hartsfield and Ringel [7] was first introduced an antimagic labeling, which is defined as a bijection $f: E \rightarrow\{1,2, \ldots,|E|\}$. For each vertex $u \in V(G)$, the weight $w(u)=\sum_{e \in E(u)} f(e)$, where $E(u)$ is the set of edges incident to $u$. If $w(u) \neq w(v)$ for any two distinct vertices $u$ and $v \in V(G)$, then $f$ is called an antimagic labeling of $G$. A graph $G$ is called antimagic if $G$ has antimagic labeling. Hartsfield and Ringel's [7] conjectured that every connected graph with at least three vertices admits antimagic labeling. They also made a weak conjecture that every tree with at least three vertices admits an antimagic labeling. These two conjectures were partially shown to be true by several authors, but they are still unsolved. For the best and most interesting results were obtained so far, one can see [10] for trees and [5] for general graphs. Also, for a detailed and interesting review on these conjectures, one can see chapter 6 of [6].

Arumugam et al.[1] posed a new definition as a relaxation of the notion of antimagic labeling. They called a bijection $f: E \rightarrow\{1,2, \ldots,|E|\}$ is a local antimagic labeling of $G$ if for any two adjacent vertices $u$ and $v$ in $V(G)$, the condition $w(u) \neq w(v)$ holds. They conjectured that every connected graph with at least three vertices admits a local antimagic labeling. This conjecture was solved partially in [3]. Finally, Haslegrave proved this conjecture by means of probabilistic tools [8]. In 2017, Bensmail, Senhaji, and Szabo Lyngsie [3] obtained the results on trees answers positively to Conjecture 2.3 raised by Arumugam et al.[1] using another aspect of neighbour-sum-distinguishing.

Based on the notion of local antimagic labeling, Arumugam et al.[1] introduced a new graph coloring parameter. We call local antimagic chromatic number $\chi_{l a}(G)$, which is defined as the minimum number of colors taken over all colorings of $G$ induced by local antimagic labelings of $G$.

In [1], they proved the local chromatic number of cycle on $n$ vertices is 3 and they observed that, if the connected graph $G$ contains a triangle $C_{3}$, then $\chi_{l a}(G) \geq 3$. Also, they proved that the local chromatic number of complete graph on $p$ vertices is $p$ colors and the local chromatic number of wheel graph $W_{n}$ is 4 , where $n$ is odd. For $n$ is even case they proved that $\chi_{l a}\left(W_{n}\right)=3$, where $n \equiv 2(\bmod 4)$. Then they obtained lower and upper 
bounds $3 \leq \chi_{l a}\left(W_{n}\right) \leq 5$ for $n \equiv 0(\bmod 4)$. Recently, in [11], the authors obtained exact value for $\chi_{l a}\left(W_{n}\right)=4$ for $n \equiv 0(\bmod 4)$.

Frucht and Harary [15] introduced the corona product of two graphs, which is defined as: The corona product of two graphs $G$ and $H$ is the graph $G \odot H$ obtained by taking one copy of $G$ along with $|V(G)|$ copies of $H$, and putting extra edges making the $i$-th vertex of $G$ adjacent to every vertex of the $i$-th copy of $H$, where $1 \leq i \leq|V(G)|$. In [2] Arumugam, Lee, Premalatha and Wang completely determined the local antimagic chromatic number $\chi_{l a}\left(G \odot \overline{K_{m}}\right)$ for the corona product of a graph $G$ with the null graph $\overline{K_{m}}$ on $m \geq 1$ vertices (or complement graph of $K_{m}$ ), when $G$ is path $P_{n}$, cycle $C_{n}$, and complete graph $K_{n}$.

Arumugam et al.[1], Shaebani [9] and Lau et al.[11, 14] studied independently, the local antimagic chromatic number for the join graphs, which is defined as: Let $G_{1}$ and $G_{2}$ be two vertex disjoint graphs. The join graph of $G_{1}$ and $G_{2}$, denoted by $G_{1} \vee G_{2}$, is the graph whose vertex set is $V\left(G_{1}\right) \cup V\left(G_{2}\right)$ and its edge set equals $E\left(G_{1}\right) \cup E\left(G_{2}\right) \cup\{a b: a \in$ $V\left(G_{1}\right)$ and $\left.b \in V\left(G_{2}\right)\right\}$.

Arumugam et al.[1] obtained lower and upper bounds for the join graph $G_{1} \vee G_{2}$. Recently, the author Shaebani [9] obtained counterexamples to a Theorem 2.16 [1] which asserts that if a graph $G$ has at least four vertices, then $\chi_{l a}(G)+1 \leq \chi_{l a}\left(G \vee \overline{K_{2}}\right)$, where $\overline{K_{2}}$ is the complement graph of a complete graph with two vertices. In this regard, the author Shaebani [9] proved that if $n$ is odd and $n+1$ is not divisible by 3 , then $\chi_{l a}\left(K_{1, n} \vee \overline{K_{2}}\right)=$ 3. Moreover, Lau et al.[11, 14] also studied the local antimagic chromatic number for join graphs independently.

In this paper, we investigate the local antimagic chromatic number for wheel related graphs.

\section{Local Chromatic Number of Helm graph}

In [12], Ayel and Favaron introduced a Helm graph, which is defined as: The helm graph is the graph obtained from a wheel graph $W_{n}$ on $n+1$ vertices by adjoining a pendant edge at each node of the cycle and $H_{n}$ denotes it.

Krishnaa [13] studied some helm related graphs that admit antimagic labeling. In this section, we study the local antimagic vertex coloring of the same graph $H_{n}$. 
Theorem 2.1. For the graph $H_{n}, 3 \leq n \leq 5$, we have

$$
\chi_{l a}\left(H_{n}\right)= \begin{cases}6, & n=3,4 \\ 8, & n=5 .\end{cases}
$$

Proof. Let $G \cong H_{n}$ be the helm graph and let $V(G)=\left\{c \cup v_{i} \cup u_{i}, 1 \leq\right.$ $i \leq n\}$ and $E(G)=\left\{c v_{i} \cup v_{i} u_{i}, 1 \leq i \leq n\right\} \cup\left\{v_{n} v_{1} \cup v_{i} v_{i+1}, 1 \leq i \leq n-1\right\}$. Then $|V(G)|=p=2 n+1$ and $|E(G)|=q=3 n$.

Case 1: $n=3$

Suppose $H_{3}$ admits a local antimagic labeling $f$. Since every pendant vertex received a new color, it follows that the pendant vertices $u_{1}, u_{2}$ and $u_{3}$ have received the colors $w_{1}, w_{2}$ and $w_{3}$. Since $1 \leq w\left(u_{i}\right) \leq 9$ and the minimum possible vertex weight of $v_{i}$ is 10 , we get $w\left(u_{i}\right) \neq w\left(v_{i}\right), i=1,2,3$ and hence the vertex $v_{1}, v_{2}$ and $v_{3}$ are received new colors $w_{4}, w_{5}$ and $w_{6}$. Thus $\chi_{l a}\left(H_{3}\right) \geq 6$. So, for proving $\chi_{l a}\left(H_{3}\right)=6$, it suffices to provide a local antimagic labeling of $H_{3}$ that induces a local antimagic vertex coloring using exactly six colors. Now, we define bijection $f: E\left(H_{3}\right) \rightarrow\{1,2,3, \ldots, 9\}$ by

$$
\begin{aligned}
& f\left(c v_{i}\right)=i, \quad i=1,2,3 \\
& f\left(v_{i} u_{i}\right)=7-i, \quad i=1,2,3 \\
& f\left(v_{1} v_{2}\right)=8, \quad f\left(v_{2} v_{3}\right)=7, \quad f\left(v_{1} v_{3}\right)=9 .
\end{aligned}
$$

In this case, we have $w(c)=w\left(u_{1}\right)=6, w\left(u_{2}\right)=5, w\left(u_{3}\right)=4, w\left(v_{1}\right)=$ $24, w\left(v_{2}\right)=22, w\left(v_{3}\right)=23$. Therefore, $f$ is a local antimagic labeling of $H_{3}$ that induces a local antimagic vertex coloring using exactly six colors.

Case 2: $n=4$

Suppose $H_{4}$ admits a local antimagic labeling $f$. Since every pendant vertex received a new color, it follows that the pendant vertices $u_{1}, u_{2}, u_{3}$ and $u_{4}$ are received the colors $w_{1}, w_{2}, w_{3}$ and $w_{4}$. Let $e=c v_{1}$ or $e=v_{1} u_{1}$ or $v_{1} v_{2}$ in $E\left(H_{4}\right)$. For any of $v_{1}, v_{2}, v_{3}, v_{4}, c$ to receive a color less than or equal to 12 , the four incident edges must include labels 1 and 2. This means that at most one of them can receive such a color, and therefore there are some two adjacent vertices which receive new colors $w_{5}, w_{6}$ greater than 12 . Thus $\chi_{l a}\left(H_{4}\right) \geq 6$. So, for proving $\chi_{l a}\left(H_{4}\right)=6$, it suffices to provide a local antimagic labeling of $H_{4}$ that induces a local antimagic vertex coloring using exactly six colors. Now, we define bijection $f: E\left(H_{4}\right) \rightarrow\{1,2,3, \ldots, 12\}$ by

$$
\begin{array}{clll}
f\left(c v_{1}\right)=1, & f\left(c v_{2}\right)=3, & f\left(c v_{3}\right)=2, & f\left(c v_{4}\right)=4, \\
f\left(v_{1} u_{1}\right)=9, & f\left(v_{2} u_{2}\right)=12, & f\left(v_{3} u_{3}\right)=10, & f\left(v_{4} u_{4}\right)=11 \\
f\left(v_{1} v_{2}\right)=6, & f\left(v_{2} v_{3}\right)=7, & f\left(v_{3} v_{4}\right)=5, & f\left(v_{1} v_{4}\right)=8
\end{array}
$$


In this case, we have $w(c)=w\left(u_{3}\right)=10, w\left(u_{1}\right)=9, w\left(u_{2}\right)=12, w\left(u_{4}\right)=$ $11, w\left(v_{1}\right)=w\left(v_{3}\right)=24, w\left(v_{2}\right)=w\left(v_{4}\right)=28$. Therefore, $f$ is a local antimagic labeling of $H_{4}$ that induces a local antimagic vertex coloring using exactly six colors.

Case 3: $n=5$

Suppose $H_{5}$ admits a local antimagic labeling $f$. Since every pendant vertex received a new color, it follows that the pendant vertices $u_{1}, u_{2}, u_{3}, u_{4}$ and $u_{5}$ are received the colors $w_{1}, w_{2}, w_{3}, w_{4}$ and $w_{5}$. Let $e=c v_{1}$ or $v_{1} u_{1}$ or $v_{1} v_{2}$ in $E\left(H_{5}\right)$. If $f(e)=15$ then the incident vertex $v_{1}$ received a new color $w_{6}$.

If $f\left(e=c v_{1}\right)=15$ then the vertex $c$ received a color $w(c)=w\left(u_{i}\right)$ or a new color $w_{7}$. If $w(c)=w\left(u_{i}\right)$ for any $i$, then $w(c)=15$ because the minimum possible vertex weight of $c$ is 15 and $q=15$. If additionally $w\left(v_{2}\right)=w\left(u_{i}\right) \in\{10,11,12,13,14\}-w\left(u_{2}\right)$, then $f\left(v_{2} u_{2}\right) \in\{6,7,8,9\}$. If $f\left(v_{2} u_{2}\right) \in\{6,7,8,9\}$ then the vertex $v_{2}$ weight is $w\left(v_{2}\right) \neq q$ and hence the vertex $v_{2}$ received a new color $w_{7}$. Suppose $w\left(v_{3}\right)=w_{6}$ and $w\left(v_{4}\right)=w_{7}$. Then the vertex $v_{5}$ must receive a new color $w_{8}$. If $w(c)=w_{7}$ then the adjacent vertex of $v_{1}$ is $v_{2}$ received a new color $w_{8}$.

If $f\left(e=v_{1} u_{1}\right)=15$ then the vertex $c$ received a color $w\left(u_{1}\right)=15$ and hence $w(c)=w\left(u_{1}\right)=15$. Therefore, the edges $\left\{c v_{i}, 1 \leq i \leq 5\right\}$ are received the labels from $\{1,2,3,4,5\}$. Then the vertex $v_{2}$ adjacent vertices are received the minimum possible labels are $\{1,6,7,8\}$, this sum gives the minimum possible weight of a vertex $v_{2}$. Therefore, the vertex $v_{2}$ received a new color $w_{7}$. Suppose $w\left(v_{3}\right)=w_{6}$ and $w\left(v_{4}\right)=w_{7}$. Then the vertex $v_{5}$ must receive a new color $w_{8}$.

If $f\left(e=v_{1} v_{2}\right)=15$ then the vertex $v_{2}$ received a new color $w_{7}$. Suppose $w\left(v_{3}\right)=w_{6}$ or $w\left(u_{i}\right), i \neq 3$ and $w\left(v_{4}\right)=w_{7}$ or $w\left(u_{i}\right), i \neq 4$. Then the vertex $v_{5}$ must receive a new color $w_{8}$. Hence $\chi_{l a}\left(H_{5}\right) \geq 8$.

So, for proving $\chi_{l a}\left(H_{5}\right)=8$, it suffices to provide a local antimagic labeling of $H_{5}$ that induces a local antimagic vertex coloring using exactly eight colors. Now, we define bijection $f: E\left(H_{5}\right) \rightarrow\{1,2,3, \ldots, 15\}$ by

$$
\begin{gathered}
f\left(c v_{1}\right)=1, \quad f\left(c v_{2}\right)=4, \quad f\left(c v_{3}\right)=2, \quad f\left(c v_{4}\right)=5, \quad f\left(c v_{5}\right)=3, \\
f\left(v_{1} u_{1}\right)=11, \quad f\left(v_{2} u_{2}\right)=13, \quad f\left(v_{3} u_{3}\right)=12, \quad f\left(v_{4} u_{4}\right)=14, \quad f\left(v_{5} u_{5}\right)=15, \\
f\left(v_{1} v_{2}\right)=7, \quad f\left(v_{2} v_{3}\right)=9, \quad f\left(v_{3} v_{4}\right)=6, \quad f\left(v_{4} v_{5}\right)=8, \quad f\left(v_{1} v_{5}\right)=10 .
\end{gathered}
$$

In this case, we have $w(c)=w\left(u_{5}\right)=15, w\left(u_{1}\right)=11, w\left(u_{2}\right)=13, w\left(u_{3}\right)=$ $12, w\left(u_{4}\right)=14, w\left(v_{1}\right)=w\left(v_{3}\right)=29, w\left(v_{2}\right)=w\left(v_{4}\right)=33, w\left(v_{5}\right)=36$. Therefore, $f$ is a local antimagic labeling of $H_{5}$ that induces a local antimagic vertex coloring using exactly eight colors. 
Theorem 2.2. Let $H_{n}, n \geq 6$ be a helm graph. Then $\chi_{l a}\left(H_{n}\right)=n+3$.

Proof. Let $H_{n}, n \geq 6$ be a helm graph and let $V\left(H_{n}\right)=\left\{c \cup v_{i} \cup u_{i}, 1 \leq i \leq n\right\}$ and $E\left(H_{n}\right)=\left\{c v_{i} \cup v_{i} u_{i}, 1 \leq i \leq\right.$ $n\} \cup\left\{v_{n} v_{1}\right\} \cup\left\{v_{i} v_{i+1}, 1 \leq i \leq n-1\right\}$. Then $\left|V\left(H_{n}\right)\right|=p=2 n+1$ and $\left|E\left(H_{n}\right)\right|=q=3 n$.

Suppose $H_{n}$ admits a local antimagic labeling $f$. Since every pendant vertex received a new color, it follows that the pendant vertices $u_{i}, 1 \leq i \leq n$ are received the colors $w_{i}, 1 \leq i \leq n$. Clearly, the minimum possible weight of a vertex $c$ is $w(c) \geq \frac{n(n+\overline{1})}{2}>q$. Therefore, the vertex $c$ received a new color $w_{n+1}$. Let $e=c v_{1}$ or $v_{1} u_{1}$ or $v_{1} v_{2}$ in $E\left(H_{n}\right)$. If $f\left(e=v_{1} u_{1}\right)=q$ or $f\left(e=c v_{1}\right)=q$ then the vertex $v_{1}$ received a new color $w_{n+2}$. Let $e^{\prime}=v_{1} v_{2} \in E\left(H_{n}\right)$. Then the vertex $v_{2}$ weight $w\left(v_{2}\right) \in W$ or $W^{\prime}=W-\{q\}$ and $w\left(v_{2}\right) \notin W$ or $W^{\prime}=W-\{q\}$, where $W=\{10,11,12, \ldots, q-1, q\}$. If the vertex $v_{2}$ weight $w\left(v_{2}\right) \notin W$ or $W^{\prime}=W-\{q\}$ then the vertex $v_{2}$ received a new color $w_{n+3}$ and hence $\chi_{l a}\left(H_{n}\right) \geq n+3$.

Let $S$ be the set of $v_{i}$ that have weight $w_{n+2}$, and $T$ be the remainder. Since no two vertices in $S$ are adjacent, $T$ contains $(n+k) / 2$ vertices for some $k \geq 0$, and covers all the edges of the main cycle together with at least $n+k$ other edges. So the sum of weights of vertices in $T$ is at least

$$
1+2+3+\ldots+2 n+k=\frac{(2 n+k)(2 n+k+1)}{2} \geq(n+k)(2 n+1) .
$$

However, the maximum possible sum of weights of vertices in $T$ if all these are in $W$ or $W^{\prime}$ is $3 n(n+k) / 2$. Therefore at least one of these vertices has a new weight $w_{n+3}$. Thus $\chi_{l a}\left(H_{n}\right) \geq n+3$.

So, for proving $\chi_{l a}\left(H_{n}\right)=n+3$ it suffices to provide a local antimagic labeling of $H_{n}$ that induces a local antimagic vertex coloring using exactly $n+3$ colors.

For $n \geq 6$, define $f_{1}: E\left(H_{n}\right) \rightarrow\{1,2,3, \ldots, q\}$ by

$$
\begin{aligned}
f_{1}\left(c v_{i}\right) & = \begin{cases}\frac{n+3}{2}, & i=1, n \text { is odd } \\
\frac{n+2}{2}, & i=1, n \text { is even } \\
\frac{3(2 n+1)-i}{2}, & i \text { is odd, } 3 \leq i \leq n \\
\frac{2(n+2)+i}{2}, & i \text { is even, } 2 \leq i \leq n\end{cases} \\
f_{1}\left(v_{i} v_{i+1}\right) & = \begin{cases}\frac{i+1}{2}, & i \text { is odd }, 1 \leq i \leq n-1 \\
\frac{2(n+3)-i}{2}, & i \text { is even, } 2 \leq i \leq n-1\end{cases}
\end{aligned}
$$




$$
\begin{aligned}
& f_{1}\left(v_{n} v_{1}\right)= \begin{cases}\frac{n+1}{2}, & n \text { is odd } \\
\frac{n+6}{2}, & n \text { is even }\end{cases} \\
& f_{1}\left(v_{i} u_{i}\right)= \begin{cases}\frac{n+5}{2}, & i=1, n \text { is odd } \\
\frac{\frac{n+4}{2},}{\frac{4 n+i+1}{2},}, & i \text { is odd, } n \text { is odd, } 3 \leq i \leq n \\
\frac{4(n+1)-i}{2}, & i \text { is even, } n \text { is odd, } 2 \leq i \leq n-1 \\
\frac{4 n+i+3}{2}, & i \text { is odd, } n \text { is even, } 3 \leq i \leq n-1 \\
\frac{2(2 n+3)-i}{2}, & i \text { is even, } n \text { is even, } 2 \leq i \leq n\end{cases}
\end{aligned}
$$

Then the weights of vertices are,

$$
\begin{aligned}
& w_{1}\left(v_{i}\right)= \begin{cases}\frac{3 n+11}{2}, & i=1, n \text { is odd } \\
\frac{3 n+14}{2}, & i=1, n \text { is even } \\
6(n+1), & i \text { is odd, } n \text { is odd, } 3 \leq i \leq n \\
4 n+7, & i \text { is even, } n \text { is odd }, 2 \leq i \leq n-1 \\
6 n+7, & i \text { is odd, } n \text { is even, } 3 \leq i \leq n-1 \\
4(n+2), & i \text { is even, } n \text { is even, } 2 \leq i \leq n\end{cases} \\
& w_{1}(c)= \begin{cases}2 n^{2}, & n \text { is odd } \\
\frac{n(4 n-1)}{2}, & n \text { is even }\end{cases} \\
& w_{1}\left(u_{i}\right)=f_{1}\left(v_{i} u_{i}\right), 1 \leq i \leq n
\end{aligned}
$$

Hence, $\chi_{l a}\left(H_{n}\right) \leq n+3$. Therefore, $f_{1}$ is a local antimagic labeling of $H_{n}$ that induces a local antimagic vertex coloring using exactly $n+3$ colors.

From Theorem 2.1 and Theorem 2.2, we obtain the following theorem.

Theorem 2.3. Let $H_{n}, n \geq 3$ be a helm graph. Then

$$
\chi_{l a}\left(H_{n}\right)= \begin{cases}n+2, & n=4 \\ n+3, & n \neq 4\end{cases}
$$

\section{Local Chromatic Number for Some Wheel Related Graphs}

In this section, we determine the local vertex antimagic chromatic number for wheel related graphs $W_{n}^{m}$, where $m$ is a fixed positive integer.

Definition 3.1. Let $W_{n}$ be a wheel graph on $n+1$ vertices. A graph $W_{n}^{m}$ is obtained from $W_{n}$ by attaching $m$ pendant vertices to any arbitrary vertex $v \neq c$ of $W_{n}$, where $c$ is the central vertex of $W_{n}$. 
Now, we consider the graph $W_{n}^{m}$, with two different $m$ values $m_{1}=$ $t+\frac{n(n-3)}{2}, t \geq 0$ and less than $m_{1}$, that is, $\frac{n(n-3)}{2}-t, t \geq 1$. The following theorem gives the exact local chromatic number of $W_{n}^{m}, m=m_{1}$ is $m_{1}+$ 1 ,for $n \geq 7$ and for every $t \geq 0$.

Theorem 3.2. Let $W_{n}^{m_{1}}$ be a graph with $n \geq 7, t \geq 0$ and $m_{1}=t+\frac{n(n-3)}{2}$. Then $\chi_{l a}\left(W_{n}^{m_{1}}\right)=m_{1}+1$.

Proof. Let $W_{n}^{m_{1}}$ be a graph with $n \geq 7$ and $m_{1}=t+\frac{n(n-3)}{2}$. Let $V\left(W_{n}^{m_{1}}\right)=\left\{c \cup v_{i} \cup u_{k}, 1 \leq i \leq n, 1 \leq k \leq m_{1}\right\}$ and $E\left(W_{n}^{m_{1}}\right)=\left\{c v_{i} \cup\right.$ $\left.v_{n} u_{k}, 1 \leq i \leq n, 1 \leq k \leq m_{1}\right\} \cup\left\{v_{i} v_{i+1} \cup v_{n} v_{1}, 1 \leq i \leq n-1\right\}$ be the vertex set and edge set of $W_{n}^{m_{1}}$. Then $\left|V\left(W_{n}^{m_{1}}\right)\right|=m_{1}+n+1$ and $\left|E\left(W_{n}^{m_{1}}\right)\right|=$ $q=m_{1}+2 n$.

Since every pendant vertex receives a different color, and $v_{n}$ receives a higher color than any of them. We get $\chi_{l a}\left(W_{n}^{m_{1}}\right) \geq m_{1}+1$. So, for proving $\chi_{l a}\left(W_{n}^{m_{1}}\right)=m_{1}+1$ it suffices to provide a local antimagic labeling of $W_{n}^{m_{1}}$ that induces a local antimagic vertex coloring using exactly $m_{1}+1$ colors. Now, we define $f_{2}: E\left(W_{n}^{m_{1}}\right) \rightarrow\left\{1,2,3, \ldots, m_{1}+2 n\right\}$ by

$$
\begin{gathered}
f_{2}\left(c v_{i}\right)= \begin{cases}n+1-i, & n \text { is even, } i \text { is odd, } 1 \leq i \leq n-1 \\
n-1-i, & n \text { is even, } i \text { is even, } 2 \leq i \leq n-2 \\
n-1, & n \text { is even, } i=n \\
n-1-i, & n \text { is odd, } i \text { is odd, } 1 \leq i \leq n-2 \\
n, & n \text { is odd, } i=n \\
n+1-i, & n \text { is odd, } i \text { is even, } 1 \leq i \leq n-1\end{cases} \\
f_{2}\left(v_{n} v_{1}\right)=n+1 \\
f_{2}\left(v_{n} u_{k}\right)=2 n+k, 1 \leq k \leq m_{1}
\end{gathered}
$$

Then the vertex weights are 


$$
\begin{aligned}
& w_{2}(c)=w_{2}\left(u_{\frac{n(n-3)}{2}}\right)=\frac{n(n+1)}{2} \\
& w_{2}\left(v_{i}\right)= \begin{cases}\frac{7 n+4}{2}, & n \text { is even, } i \text { is odd, } 1 \leq i \leq n-1 \\
\frac{7 n}{2}, & n \text { is even, } i \text { is even, } 2 \leq i \leq n-2 \\
\frac{7 n+1}{2}, & n \text { is odd, } i \text { is odd, } 1 \leq i \leq n-2 \\
\frac{7 n+5}{2}, & n \text { is odd, } i \text { is even, } 2 \leq i \leq n-1 \\
\frac{8 n+4 n m_{1}+m_{1}\left(m_{1}+1\right)}{2}, & n \text { is even, } i=n \\
\frac{7 n+3+4 n m_{1}+m_{1}\left(m_{1}+1\right)}{2}, & n \text { is odd, } i=n\end{cases} \\
& w_{2}\left(u_{k}\right)=2 n+k, 1 \leq k \leq m_{1} .
\end{aligned}
$$

Hence, $\chi_{l a}\left(W_{n}^{m_{1}}\right) \leq m_{1}+1$. Therefore, $f_{2}$ is a local antimagic labeling of $W_{n}^{m_{1}}$ that induces a local antimagic vertex coloring using exactly $m_{1}+1$ colors.

From Theorem 3.2, we observe the local chromatic number for the graph $W_{n}^{m}$, with $m<m_{1}$ is greater than $m+1$. We consider $m=\frac{n(n-3)}{2}-t<$ $m_{1}, t \geq 1$. Since all the pendant vertices of $W_{n}^{m}$ received new color say $w_{i}, 1 \leq i \leq m$, and these pendant vertices adjacent to a vertex $v$ also received a new color $w_{m+1}$. Clearly, the minimum possible central vertex weight is $w(c)=\frac{n(n+1)}{2}>q=m+2 n, n \geq 4$. Therefore, the central vertex $c$ received a new color $w_{m+2}$. Hence $\chi_{l a}\left(W_{n}^{m}\right) \geq m+2$ and gives the following remark.

Remark 3.3. Let $W_{n}^{m}, n \geq 4$ be a graph with $m=\frac{n(n-3)}{2}-t, t \geq 1$. Then $\chi_{l a}\left(W_{n}^{m}\right) \geq m+2$.

Definition 3.4. Let $W_{n}^{m}, n \geq 5$ be a wheel graph on $n+1$ vertices. A graph $\widehat{W_{n}^{m}}$ is obtained from $W_{n}$ by attaching $m_{2}=\left\lceil\frac{n-4}{2}\right\rceil$, pendant vertices in every vertex $v \neq c$ of $W_{n}$.

Theorem 3.5. Let ${\widehat{W_{n}^{m}}}_{2}$ be a graph, where $n \geq 5$ and $m_{2}=\left\lceil\frac{n-4}{2}\right\rceil$. Then $\chi_{l a}\left({\widehat{W_{n}^{m}}}^{2}\right)=m_{2} n+3$.

Proof. Let ${\widehat{W_{n}^{m}}}_{2}$ be the graph and let $V\left({\widehat{W_{n}^{m}}}^{2}\right)=\left\{c \cup v_{i} \cup u_{i}^{k}, 1 \leq i \leq\right.$ $\left.n, 1 \leq k \leq m_{2}\right\}$ and $E\left({\widehat{W_{n}^{m}}}_{2}\right)=\left\{c v_{i} \cup v_{i} v_{i+1} \cup v_{n} v_{1} \cup v_{i} u_{i}^{k}, 1 \leq i \leq n, 1 \leq\right.$ $\left.k \leq m_{2}\right\}$. Then $\left|V\left(\widehat{W_{n}^{m}}\right)\right|=n\left(m_{2}+1\right)+1$ and $\left|E\left({\widehat{W_{n}^{m}}}_{2}\right)\right|=n\left(m_{2}+2\right)$.

Since every pendant vertex received a new color it follows that, the vertices $u_{i}^{k}$ are received the colors $w_{t}, 1 \leq t \leq m_{2} n$. If $n$ is even, then the minimum possible weight of the central vertex $c$ is $w(c)=\frac{n(n+1)}{2}>q$ and hence the vertex $c$ received a new color $w_{m_{2} n+1}$. Let $e \in E\left(\widehat{W_{n}^{m}}\right)$. If 
$f\left(e=v_{1} v_{2}\right)=q$ then its incident vertex $v_{1}$ received a new color $w_{m_{2} n+2}$ and hence the vertex $v_{2}$ received a new color $w_{m_{2} n+3}$. A similar argument applies for the case of $f\left(e=v_{1} u_{1}\right)=q$ or $f\left(e=c v_{1}\right)=q$ in Theorem 2.2 with substituting $u_{1}$ by $u_{1}^{k}$ and $q=n\left(m_{2} n+2\right)$. Thus the vertex $v_{2}$ received a new color $w_{m_{2} n+3}$.

If $n$ is odd, the minimum possible weight of the central vertex is $q$. If it receives a higher weight, we proceed as above. If it receives weight $q$ then we necessarily have $f\left(c v_{1}\right), \ldots, f\left(c v_{n}\right)$ being $1,2, \ldots, n$ in some order. It follows that $w\left(v_{i}\right)>\left(d\left(v_{i}\right)-1\right)(n+1)+1>q$, so all colors used on $\left\{v_{1}, \ldots, v_{n}\right\}$ are new. Since it is an odd cycle, there are at least three new colors used. Thus, where $d\left(v_{i}\right)$ is degree of the vertex $v_{i} \cdot \chi_{l a}\left({\widehat{W_{n}^{m}}}^{2}\right) \geq m_{2} n+3$.

So, for proving $\chi_{l a}\left({\widehat{W_{n}^{m}}}^{2}\right)=m_{2} n+3$ it suffices to provide a local antimagic labeling of $\widehat{W}_{n}^{m}$ that induces a local antimagic vertex coloring using exactly $m_{2} n+3$ colors. If $m_{2}=1$ then $n=5$ and 6 . Clearly, the graph $\widehat{W_{5}^{1}} \cong H_{5}$ and $\widehat{W_{6}^{1}} \cong H_{6}$ and by Theorem 2.3 , we get $\chi_{l a}\left(\widehat{W_{n}^{m}}\right)=n+3$, where $n=5,6$.

If $m_{2}=2$ then we get $n=7$ and 8 . For $n=7$, we define $f: E\left(\widehat{W_{7}^{2}}\right) \rightarrow$ $\{1,2,3, \ldots, 28\}$ by

$$
\begin{aligned}
& f\left(c v_{i}\right)=i, 1 \leq i \leq 7 \\
& f\left(v_{i} v_{i+1}\right)=15-i, 1 \leq i \leq 6 \\
& f\left(v_{7} v_{1}\right)=8, \quad f\left(v_{1} u_{1}^{1}\right)=27, \quad f\left(v_{1} u_{1}^{2}\right)=28 \\
& f\left(v_{i} u_{i}^{k}\right)= \begin{cases}14+\frac{i}{2}, \quad i=2,4,6 \text { and } k=1 \\
17+\frac{i}{2}, \quad i=2,4,6 \text { and } k=2 \\
20+\frac{i-1}{2}, \quad i=3,5,7 \text { and } k=1 \\
23+\frac{i-1}{2}, \quad i=3,5,7 \text { and } k=2 .\end{cases} \\
& f\left(v_{i} u_{i}^{k}\right)=\left\{\begin{array}{l}
\text { and } \\
\text { ind }
\end{array}\right.
\end{aligned}
$$

In this case, we have $w(c)=w\left(u_{1}^{2}\right)=28, w\left(u_{i}^{k}\right)=f\left(v_{i} u_{i}^{k}\right), w\left(v_{1}\right)=$ $78, w\left(v_{3}\right)=w\left(v_{5}\right)=w\left(v_{7}\right)=73, w\left(v_{2}\right)=w\left(v_{4}\right)=w\left(v_{6}\right)=62$. Therefore, $f$ is a local antimagic labeling of $\widehat{W_{7}^{2}}$ that induces a local antimagic vertex coloring using exactly 17 colors.

For $n=8$, we define $f: E\left(\widehat{W_{8}^{2}}\right) \rightarrow\{1,2,3, \ldots, 32\}$ by

$$
\begin{aligned}
& f\left(c v_{i}\right)=i, 1 \leq i \leq 8 \\
& f\left(v_{i} v_{i+1}\right)=17-i, 1 \leq i \leq 7 \\
& f\left(v_{8} v_{1}\right)=9 \quad f\left(v_{1} u_{1}^{1}\right)=20, \quad f\left(v_{1} u_{1}^{2}\right)=24 \\
& f\left(v_{i} u_{i}^{k}\right)= \begin{cases}25+\frac{i-2}{2}, & i=2,4,6,8 \text { and } k=1 \\
29+\frac{i-2}{2}, & i=2,4,6,8 \text { and } k=2 \\
16+\frac{i-1}{2}, & i=3,5,7 \text { and } k=1 \\
20+\frac{i-1}{2}, & i=3,5,7 \text { and } k=2 .\end{cases} \\
& f\left(v_{i} u_{i}^{k}\right)=\left\{\begin{array}{l}
\text { a } \\
\text { ind }
\end{array}\right.
\end{aligned}
$$


In this case, we have $w(c)=36, w\left(u_{i}^{k}\right)=f\left(v_{i} u_{i}^{k}\right), w\left(v_{1}\right)=w\left(v_{3}\right)=$ $w\left(v_{5}\right)=w\left(v_{7}\right)=70, w\left(v_{2}\right)=w\left(v_{4}\right)=w\left(v_{6}\right)=w\left(v_{8}\right)=87$. Therefore, $f$ is a local antimagic labeling of $\widehat{W_{8}^{2}}$ that induces a local antimagic vertex coloring using exactly 19 colors.

For $n \geq 9$, we define a labeling $f_{3}: E\left({\widehat{W_{n}^{m}}}^{2}\right) \rightarrow\left\{1,2,3, \ldots, n m_{2}+2 n\right\}$ as follows:

Case 1: $m_{2} \geq 3$ is odd

$$
\begin{aligned}
& f_{3}\left(c v_{i}\right)= \begin{cases}\frac{i+1}{2}, & n \text { is odd, } i \text { is odd, } 1 \leq i \leq n-2 \\
\frac{2 n-i}{2}, & n \text { is odd, } i \text { is even, } 2 \leq i \leq n-1 \\
n, & n \text { is odd, } i=n \\
\frac{i+1}{2}, & n \text { is even, } i \text { is odd, } 1 \leq i \leq n-1 \\
\frac{2 n+2-i}{2}, & n \text { is even, } i \text { is even, } 2 \leq i \leq n\end{cases} \\
& f_{3}\left(v_{i} v_{i+1}\right)= \begin{cases}\frac{3 n+i}{2}, & n \text { is odd, } i \text { is odd, } 1 \leq i \leq n-2 \\
\frac{3 n+1-i}{2}, & n \text { is odd, } i \text { is even, } 2 \leq i \leq n-1 \\
\frac{2 n+1+i}{2}, & n \text { is even, } i \text { is odd, } 1 \leq i \leq n-1 \\
\frac{4 n-i}{2}, & n \text { is even, } i \text { is even, } 2 \leq i \leq n-2\end{cases} \\
& f_{3}\left(v_{n} v_{1}\right)=2 n \\
& f_{3}\left(v_{i} u_{i}^{1}\right)= \begin{cases}\frac{2 n+1,}{\frac{5 n+2-i}{2},}, & n \text { is odd, } i=1 \\
\frac{5 n-1+i}{2}, & n \text { is odd, } i \text { is odd }, 3 \leq i \leq n-2 \\
3 n, & n \text { is odd, } i=n \\
\frac{5 n+1-i}{2}, & n \text { is even, } i \text { is odd, } 1 \leq i \leq n-1 \\
\frac{5 n+2+i}{2}, & n \text { is even, } i \text { is even, } 2 \leq i \leq n-2 \\
\frac{5 n+2}{2}, & n \text { is even, } i=n\end{cases} \\
& f_{3}\left(v_{i} u_{i}^{k}\right)= \begin{cases}3 n+\frac{n-1}{2}(k-2)+\left(\frac{i+1}{2}\right), & n \text { is odd, } i \text { is odd, } k \text { is even, } \\
3 n+\frac{n-1}{2}(k-1)-\left(\frac{i-1}{2}\right), & 1 \leq i \leq n-2,2 \leq k \leq m_{2} \\
3 n+\frac{n-1}{2}\left[\left(m_{2}-1\right)+(k-2)\right]+\left(\frac{i}{2}\right), & n \text { is odd, } i \text { is odd, } k \text { is odd, } \\
& n \text { is odd, } i \text { is even, } k \text { is even, } \\
3 n+\frac{n-1}{2}\left[\left(m_{2}-1\right)+(k-1)\right]-\left(\frac{i-2}{2}\right), & n \text { is odd, } i \text { is even, } k \text { is odd, } \\
3 n+2\left(m_{2}-1\right) \frac{n-1}{2}+(k-1), & 1 \leq i \leq n-1,3 \leq k \leq m_{2} \\
3 \text { is odd, } i=n, 2 \leq k \leq m_{2}\end{cases}
\end{aligned}
$$




$$
f_{3}\left(v_{i} u_{i}^{k}\right)= \begin{cases}3 n+\frac{n}{2}(k-2)+\left(\frac{i+1}{2}\right), & n \text { is even, } i \text { is odd, } k \text { is even, } \\ 3 n+\frac{n}{2}(k-1)-\left(\frac{i-1}{2}\right), & 1 \leq i \leq n-1,2 \leq k \leq m_{2} \\ 3 n+\frac{n}{2}\left[\left(m_{2}-1\right)+(k-2)\right]+\left(\frac{i}{2}\right), & n \text { is even, } i \text { is odd, } k \text { is odd, } \\ & 1 \leq i \leq n-1,3 \leq k \leq m_{2} \\ 3 n+\frac{n}{2}\left[\left(m_{2}-1\right)+(k-1)\right]-\left(\frac{i-2}{2}\right), & 1 \leq i \leq n, 2 \leq k \leq m_{2} \\ & 1 \leq i \leq n, 3 \leq k \leq m_{2} .\end{cases}
$$

Case 2: $m_{2} \geq 4$ is even

$$
\begin{aligned}
& f_{3}\left(c v_{i}\right)=n-i+1,1 \leq i \leq n \\
& f_{3}\left(v_{i} v_{i+1}\right)=n+i+1,1 \leq i \leq n-1 \\
& f_{3}\left(v_{n} v_{1}\right)=n+1 \text {. } \\
& f_{3}\left(v_{i} u_{i}^{k}\right)= \begin{cases}\frac{7 n-i-2}{2}, & k=1, n \text { is odd, } i \text { is odd, } 1 \leq i \leq n-2 \\
\frac{5 n-i+1}{2}, & k=1, n \text { is odd, } i \text { is even, } 2 \leq i \leq n-1 \\
4 n-1, & k=1, n \text { is odd, } i=n, \\
\frac{8 n-i-3}{2}, & k=2, n \text { is odd, } i \text { is odd, } 1 \leq i \leq n-2 \\
\frac{6 n-i}{2}, & k=2, n \text { is odd, } i \text { is even, } 2 \leq i \leq n-1 \\
4 n, & k=2, n \text { is odd, } i=n .\end{cases} \\
& f_{3}\left(v_{i} u_{i}^{k}\right)= \begin{cases}4 n+\frac{n-1}{2}\left[\left(m_{2}-2\right)+(k-2)\right]-\left(\frac{i-2}{2}\right), & n \text { is odd, } i \text { is even, } k \text { is even, } \\
4 n+\frac{n-1}{2}\left[\left(m_{2}-2\right)+(k-3)\right]+\frac{i}{2}, & 2 \leq i \leq n-1,4 \leq k \leq m_{2} \\
4 n+\left(\frac{n-1}{2}\right)(k-2)-\left(\frac{i-1}{2}\right), & n \text { is odd, } i \text { is even, } k \text { is odd } \\
& 2 \leq i \leq n-1,3 \leq k \leq m_{2} \\
4 n+\left(\frac{n-1}{2}\right)(k-3)+\left(\frac{i+1}{2}\right), & n \text { is odd, } i \text { is odd, } k \text { is even, } \\
& 1 \leq i \leq n-2,4 \leq k \leq m_{2} \\
4 n+(n-1)\left(m_{2}-2\right)+k-2, & n \text { is odd, } i \text { is odd, } k \text { is odd, } \\
& 1 \leq i \leq n-2,3 \leq k \leq m_{2} \\
& n \text { is odd, } i=n, 3 \leq k \leq m_{2} .\end{cases} \\
& f_{3}\left(v_{i} u_{i}^{k}\right)= \begin{cases}\frac{7 n-i+1}{2}, & k=1, n \text { is even, } i \text { is odd, } 1 \leq i \leq n-1 \\
\frac{5 n-i}{2}, & k=1, n \text { is even, } i \text { is even, } 2 \leq i \leq n-2 \\
\frac{5 n}{2}, & k=1, n \text { is even, } i=n \\
\frac{8 n-i+1}{2}, & k=2, n \text { is even, } i \text { is odd, } 1 \leq i \leq n-1 \\
\frac{6 n-i}{2}, & k=2, n \text { is even, } i \text { is even, } 2 \leq i \leq n-2 \\
\frac{6 n}{2}, & k=2, n \text { is even, } i=n .\end{cases}
\end{aligned}
$$




$$
f_{3}\left(v_{i} u_{i}^{k}\right)= \begin{cases}4 n+\frac{n}{2}\left[\left(m_{2}-2\right)+(k-2)\right]-\left(\frac{i-2}{2}\right), & n \text { is even, } i \text { is even, } k \text { is even, } \\ 4 n+\frac{n}{2}\left[\left(m_{2}-2\right)+(k-3)\right]+\frac{i}{2}, & 2 \leq i \leq n, 4 \leq k \leq m_{2} \\ 4 n+\frac{n}{2}(k-2)-\left(\frac{i-1}{2}\right), & n \text { is even, } i \text { is even, } k \text { is odd, } \\ & 2 \leq i \leq n, 3 \leq k \leq m_{2} \\ 4 n+\frac{n}{2}(k-3)+\left(\frac{i+1}{2}\right), & n \text { is even, } i \text { is odd, } k \text { is even, } \\ & 1 \leq i \leq n-1,4 \leq k \leq m_{2} \\ & n \text { is even, } i \text { is odd, } k \text { is odd }, \\ & 1 \leq i \leq n-1,3 \leq k \leq m_{2} .\end{cases}
$$

Then the weight of the vertices are,

$$
w_{3}\left(u_{i}^{k}\right)= \begin{cases}f_{3}\left(v_{i} u_{i}^{k}\right), & n \text { is even, } 1 \leq i \leq n, 1 \leq k \leq m_{2}, \\ f_{3}\left(v_{i} u_{i}^{k}\right), & n \text { is odd, } 1 \leq i \leq n-1,1 \leq k \leq m_{2}, \\ f_{3}\left(v_{i} u_{i}^{k}\right), & n \text { is odd, } i=n, 1 \leq k \leq m_{2}-1 \\ f_{3}\left(v_{i} u_{i}^{k}\right)=w_{3}(c), & n \text { is odd, } i=n, k=m_{2} .\end{cases}
$$

If $m_{2} \geq 3$ is odd, then,

$$
w_{3}\left(v_{i}\right)= \begin{cases}A+\frac{11 n+5}{2}, & n \text { is odd, } i \text { is odd, } 1 \leq i \leq n-2 \\ B+\frac{13 n-1}{2}, & n \text { is odd, } i \text { is even, } 2 \leq i \leq n-1 \\ C+7 n+1, & n \text { is odd, } i=n \\ D+\frac{11 n+4}{2}, & n \text { is even, } i \text { is odd, } 1 \leq i \leq n-1 \\ E+\frac{13 n+4}{2}, & n \text { is even, } i \text { is even, } 2 \leq i \leq n .\end{cases}
$$

Where

$$
\begin{aligned}
& A=3 n\left(m_{2}-1\right)+\frac{1}{4} m_{2}\left(m_{2}-1\right)(n-1), \\
& B=3 n\left(m_{2}-1\right)+\frac{\left(m_{2}-1\right)}{4}\left[\left(3 m_{2}-3\right)(n-1)+2\right] \\
& C=\left(m_{2}-1\right)\left[3 n+\left(m_{2}-1\right)(n-1)\right]+\frac{1}{2} m_{2}\left(m_{2}-1\right) \\
& D=3 n\left(m_{2}-1\right)+\frac{1}{4}\left(m_{2}-1\right)\left(n m_{2}-n+2\right) \\
& E=3 n\left(m_{2}-1\right)+\frac{\left(m_{2}-1\right)}{4}\left[n\left(3 m_{2}-3\right)+2\right] .
\end{aligned}
$$

If $m_{2} \geq 4$ is even, then

$$
w_{3}\left(v_{i}\right)= \begin{cases}P+\frac{21 n-1}{2}, & n \text { is odd, } i \text { is odd, } 1 \leq i \leq n-2 \\ Q+\frac{17 n+5}{2}, & n \text { is odd, } i \text { is even, } 2 \leq i \leq n-1 \\ R+11 n+1, & n \text { is odd, } i=n \\ S+\frac{21 n+6}{2}, & n \text { is even, } i \text { is odd, } 1 \leq i \leq n-1 \\ T+\frac{17 n+4}{2}, & n \text { is even, } i \text { is even, } 2 \leq i \leq n\end{cases}
$$

Where

$$
\begin{aligned}
& P=4 n\left(m_{2}-2\right)+\frac{\left(m_{2}-2\right)}{4}\left[(n-1) m_{2}-2 n+4\right] \\
& Q=4 n\left(m_{2}-2\right)+\frac{\left(m_{2}-2\right)}{4}\left[(n-1)\left(3 m_{2}-6\right)+2\right] \\
& R=\left(m_{2}-2\right)\left[4 n+\left(m_{2}-2\right)(n-1)\right]+\frac{1}{2}\left(m_{2}-2\right)\left(m_{2}-1\right) \\
& S=4 n\left(m_{2}-2\right)+\frac{\left(m_{2}-2\right)}{4}\left[n\left(m_{2}-1\right)-n+2\right] \\
& T=4 n\left(m_{2}-2\right)+\frac{\left(m_{2}-2\right)}{4}\left[n\left(3 m_{2}-6\right)+2\right] .
\end{aligned}
$$


Clearly, any two adjacent vertices $v_{i}$ and $v_{j}$ receive different colors and hence $\chi_{l a}\left(\widehat{W_{n}^{m_{2}}}\right) \leq m_{2} n+3$. Thus $\chi_{l a}\left(\widehat{W_{n}^{m}}\right)=m_{2} n+3$.

\section{Conclusion}

We proved the local chromatic number for helm graph and wheel-related graphs $W_{n}^{m}$ with two different $m$ values. A natural open question is to extend this technique to more general graphs having exactly only one maximum degree vertex, other than the wheel graph.

\section{Acknowledgement}

The authors are thankful to the reviewer for helpful suggestions leading to substantial improvement in the presentation of the paper. R. Shankar is thanks to the Vellore Institute of Technology, Vellore for providing a Teaching Research Associate Fellowship.

\section{References}

[1] S. Arumugam, K. Premalatha, M. Bača, and A. Semaničová-Feňovčíková, "Local antimagic vertex coloring of a graph", Graphs and combinatorics, vol. 33, no. 2, pp. 275-285, 2017. doi: 10.1007/ s00373-017-1758-7

[2] K. Premalatha, S. Arumugam, Y.-C. Lee, and T.-M. Wang, "Local antimagic chromatic number of trees - I", Journal of discrete mathematical sciences and cryptography, pp. 1-12, 2020. doi: 10.1080/09720529. 2020.1772985

[3] J. Bensmail, M. Senhaji and K. Szabo Lyngsie, "On a combination of the 1-2-3 conjecture and the antimagic labelling conjecture", Discrete mathematics and theoretical computer science, vol. 19, no. 1, 2017. [On line]. Available: https:/ / bit.ly/ 3fO40kE

[4] G. Chartrand and L. Lesniak, Graphs \& Digraphs, 4th ed. Boca Raton: Chapman Hall/ CRC, 2005.

[5] T. Eccles, "Graphs of large linear size are antimagic", Journal of graph theory, vol. 81, no. 3, pp. 236-261, 2015. doi: 10.1002/ jgt.21872

[6] J. A. Gallian, "A dynamic survey of graph labeling”, The Electronic Journal of Combinatorics, \#DS6, 2020. 
[7] N. Hartsfield and G. Ringel, Pearls in graph theory, Boston: Academic Press, 1994.

[8] J. Haslegrave, "Proof of a local antimagic conjecture", Discrete mathematics \& theoretical computer science, vol. 20, no. 1, 2018.

[9] S. Shaebani, "On Local antimagic chromatic number of graphs", Journal of algebraic systems, vol. 7, no. 2, pp. 245-256, 2020. doi: 10.22044/ JAS.2019.7933.1391

[10] Y.-C. Liang, T.-L. Wong, and X. Zhu, "Anti-magic labeling of trees", Discrete mathematics, vol. 331, pp. 9-14, 2014. doi: 10.1016/j.disc. 2014.04.021

[11] G.-C. Lau, H.-K. Ng, and W.-C. Shiu, "Affirmative solutions on local antimagic chromatic number", Graphs and combinatorics, vol. 36, no. 5, pp. 1337-1354, 2020. doi: 10.1007/ s00373-020-02197-2

[12] J. Ayel and O. Favaron, "Helms are graceful" in Progress in graph theory, J. A. Bondy and U.S.R. Murty, Eds. Toronto: Academic Press, 1984, pp. 89-92.

[13] A. Krishnaa, "Formulas and algorithms of antimagic labelings of some Helm related graphs", Journal of discrete mathematical sciences and cryptography, vol. 19, no. 2, pp. 425-434, 2016. doi: 10.1080/09720529.2015.1130935

[14] G.-C. Lau, H.-K. Ng, and W.-C. Shiu, "On local antimagic chromatic number of cycle-related join graphs", Discussiones mathematicae graph theory, vol. 41, no. 1, p. 133-152, 2021. doi: 10.7151/ dmgt.2177

[15] R. Frucht and F. Harary, "On the corona of two graphs", Aequationes mathematicae, vol. 4, no. 1-2, pp. 264-264, 1970. doi: 10.1007/ bf01817769 


\section{R. Shankar}

Department of Mathematics

School of Advanced Sciences

Vellore Institute of Technology

Vellore-632 014, Tamilnadu

India

e-mail: rathinavelshankar@gmail.com

and

\section{Nalliah}

Department of Mathematics

School of Advanced Sciences

Vellore Institute of Technology

Vellore-632 014, Tamilnadu

India

e-mail: nalliahklu@gmail.com

Corresponding author 